\title{
Antimicrobial resistance pattern of faecal Escherichia coli isolates: story of antibiotic use and misuse
}

\author{
Sayal $\mathbf{P}^{1}$, Kumar $\mathbf{S}^{2}$ \\ ${ }^{1}$ Dr. Pallavi Sayal, Senior Resident, ${ }^{2}$ Dr. Surinder Kumar, Professor \& Head of Department, both authors are attached \\ with Department of Microbiology, BPS, GMC (W), Khanpur Kalan, Sonepat, Haryana, India.
}

Address for Correspondence: Dr. Pallavi Sayal, Senior Resident, BPS, GMC (W), Khanpur Kalan, Sonepat, Haryana. Email id: petalz03@gmail.com

\begin{abstract}
Introduction: Antimicrobial resistance is a major challenge of concern for treating patients. Emergence of antibiotic resistance among hospitalized patients has received considerable emphasis, while multi drug resistance also has an impact on community. It is well known that commensal faecal flora are reservoir of resistance genes, as well as site where resistance genes can be transferred. Aims \& Objectives: The aim of the present study was to determine the antimicrobial resistance pattern of Escherichia coli isolates from patients with suspected intestinal infection with and without diarrhoea attending outpatient department in tertiary care center. Material \& Methods: This retrospective short observational study was carried out on 111 non repetitive fecal samples received in microbiology laboratory. Escherichia coli (E.coli) strains isolated were evaluated for resistance pattern. Results: Among 46(41.81\%) isolated E.coli strains $84.78 \%$ isolates exhibited resistance to two or more antimicrobials, whereas $45.65 \%$ isolates were multi drug resistant. Decreased susceptibility was observed for first line antibiotics which tend to be more affordable and accessible to families, followed by $3^{\text {rd }}$ generation cephalosporin's. However, resistance among quinolones and aminoglycosides varies between 20-30\%. Imipenem was observed as most sensitive followed by Piperacillin + Tazobactum (100.00\% and 86.95\% respectively). Conclusion: Consequences of resistance are harder to define than microbiologists might wish. Some patients recover despite inadequate treatment whereas others failed to respond despite appropriate therapy. Increased morbidity and mortality are the most dramatic consequences of drug resistance.
\end{abstract}

Key words- Intestinal E.coli, Antibiotic resistance, Drug Resistance, Colonization

\section{Background}

During last several years an alarming worldwide increase in incidence of multi drug resistant bacteria (MDR) has been observed [1]. Basic mechanism of resistance is well known, but critical new aspects continue to be discovered. Factors with major implications for the emergence, dissemination, and maintenance of resistance include multidrug efflux, hyper mutability, integrons, and plasmid addiction. Antibacterial use disrupts the microbial ecology of the patient. Though correlation between in vitro resistance and treatment failure is imperfect, but resistance undoubtedly increases mortality, morbidity, and costs in many settings [2].

Manuscript received: $20^{\text {th }}$ March 2017

Reviewed: $28^{\text {th }}$ March 2017

Author Corrected: $4^{\text {th }}$ April 2017

Accepted for Publication: 12 ${ }^{\text {th }}$ April 2017
Mechanism of Multi Drug Resistance among Gram Negative Bacteria (GNB)

Acquisition of Resistance via mutation- During deoxyribonucleic acid (DNA) replication, mutations occur randomly at a frequency of $10^{-9}$ to $10^{-10}$ per gene. As a result, of uncorrected base substitution, copying errors, targets may be altered leading to druginactivation or up/down regulation of efflux and uptake pathways. Antimicrobials cause the selection of preexisting variants, once selected by this first drug, they are then primed to develop resistance to further agents. The selection or transient induction of hyper mutability may explain why variants with multiple mutations have emerged more rapidly than was predicted from laboratory studies [3]. 
Most mutations affect only a single antibacterial class, but those affecting impermeability or efflux may have a pleiotropic effect. It is best understood in Pseudomonas. aeruginosa, where mutation at mexR upregulates the mexA-mexB-oprM operon and raises the minimum inhibitory concentration (MICs) of beta-lactams (except imipenem), fluoroquinolones, tetracyclines, chloramphenicol, macrolides, various disinfectants, detergents, and organic solvents [4]. Multidrug efflux systems are also being reported in many other gram-negative bacteria, including Enterobacteriaceae. In Escherichia coli (E.coli) and among other members of Enterobacteriaceae, mutation at mar simultaneously affects the expression of $>60$ genes reducing expression of porin $\mathrm{OmpF}$ and upregulating $\mathrm{Acr} A B$, which is implicated in the efflux of beta-lactams, fluoroquinolones, chloramphenicol, tetracycline, dyes, and detergents [5].

\section{Acquisition of Resistance via DNA transfer- DNA transfer among bacteria is critical to the dissemination of resistance most often by plasmids. Within plasmids, resistance genes are often carried by transposons, which can shuttle determinants between more and less promiscuous plasmids, or into and out of the chromosome [6].}

Integrons facilitate acquisition and expression of resistance determinants behind a single promoter. They are widely distributed among gram-negative bacteria, often occurring within plasmids and transposons, and are particularly important in the dissemination of resistance genes to sulfonamides (sull), and streptomycin (aadA3). Other genes often located in integrons include those for various OXA, PSE, VIM, and IMP b-lactamases and for many aminoglycosidemodifying enzymes [7].

Many of the resistance determinants found on plasmids are believed to have originated in the chromosomes of other species, The plasmid-mediated SHV b-lactamases are derived from the chromosomal b-lactamases of Klebsiella pneumoniae; the plasmid-borne AmpC enzymes emerging in Klebsiella spp. and E. coli are chromosomal escapes from Citrobacter freundii, Hafnia alvei, Morganella morganii, and Enterobacter cloacae [8], several CTX-M cefotaximases are chromosomal escapes from Kluyvera spp [9]. Plasmids encoding the TEM plasmid-mediated b-lactamases were first recognized in 1965 but have since spread - varying with the country, unit, and species-to $30 \%-60 \%$ of clinical Enterobacteriaceae, to a few $P$. aeruginosa, and to anywhere between $1 \%-50 \%$ of Haemophilus influenzae and Neisseria gonorrhoeae isolates [10].

Resistance phenomenon is related to many other factors, including excessive antibiotic use in both human and veterinary medicine and cross transmission of resistant strains from humans to humans and from animals to humans [11]. One of the possible way for entry of various microbes could be handling of meat and meat products by adopting improper hygienic measures during handling and processing. Also, vegetables are often contaminated through insufficiently - treated water, fertilizers and by the use of biocides during cultivation. Therefore, raw meat and vegetables likely to carry large numbers of bacteria. Bacteria escaping alive through the digestive tract to the colon are often transient and resident flora have a protective effect against intruders.

The transfer of drug resistance within the gastrointestinal tract is still possible, if our food contains substantial numbers of resistant bacteria it could be an important source of resistance in fecal flora. Antibiotics often used for therapy of infected humans and animals as well as for prophylaxis and growth promotion of food producing animals. It has been suggested that resistance in bacterial populations may spread from one ecosystem to another. Thus, wild dissemination of antimicrobial resistance among bacterial populations is an increasing problem worldwide [12].

As commensal bacteria constitute as reservoir of resistance genes for potentially pathogenic bacteria, their level of resistance is considered to be a good indicator for selection pressure by antibiotic use and for resistance problems to be expected in pathogens [13].

Changes in the composition of the gut flora, can happen silently, leading to the selection of highly resistant bacteria. These resistant organisms may remain for months in the gut of the carrier without causing any symptoms or translocate through the gut epithelium, induce healthcare-associated infections, undergo crosstransmission to other individuals and cause limited outbreaks [11].

Therefore this study was designed to determine the antibiotic sensitivity pattern of $E$. coli isolates from faecal samples. 


\section{Material \& Methods}

Study design- This was a descriptive study (six months) designed to investigate the presence of drug resistance among faecal isolates. Study was carried out in the Department of Microbiology at a tertiary care hospital catering rural population. Study population includes all age group patients attending Inpatient and Outpatient department. A total of 111 non repetitive fecal samples (patients with or without diarrhoea) received in microbiology laboratory were cultured on MacConkey agar and blood agar and incubated overnight aerobically at $37^{\circ} \mathrm{C}$. Isolates were identified by conventional microbiological techniques [14].

Study organism- E. coli has been selected as study organism, as this gram negative, facultative anaerobe, rod shaped bacterium of genus Escherichia commonly found in large intestine of humans and other warmblooded animals. These strains can be commensal, existing in a symbiotic state providing resistance against pathogenic organisms or can be pathogenic and cause diseases of intestinal and extra-intestinal sites because of several virulence factors. ${ }^{14} \mathrm{E}$. coli is known for its high genetic flexibility, to acquire and transfer resistance or virulence genes from or to other strains of same species and to other genera [15]. Thus, faecal $E$. coli is regarded as a useful indicator of the spread of acquired antibiotic resistance genes in the community [16].

Antibiotic susceptibility testing- Antimicrobial sensitivity tests were carried out by Kirby-Bauer disc diffusion technique using Muller Hinton Agar as recommended by Clinical Laboratory Standard Institute (CLSI) [17]. Commercially available antibiotics discs (Hi-media, Mumbai) used were Amikacin $(30 \mu \mathrm{g})$, Gentamicin (10ug), Ampicillin $(10 \mu \mathrm{g})$, Cotrimoxazole

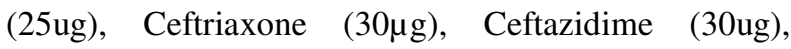
Ciprofloxacin (5ug), Imipenem (10ug), (10/30ug) and Piperacillin+ Tazobactum (100/10ug).

\section{Observations and Results}

A total of 46 biochemically confirmed isolates of E.coli were tested for their antimicrobial susceptibility pattern against 09 antimicrobial agents by disc diffusion method as per CLSI guidelines [17]. The rates of resistance observed for individual antibiotics and selected combinations of antibiotics, are shown in Table 1.

Table-1: Antimicrobial resistance pattern of E.coli isolates.

\begin{tabular}{|c|c|c|}
\hline Antimicrobial agents & $\%$ Sensitive & $\%$ Resistant \\
\hline $\begin{array}{l}\text { 1.b-lactam } \\
\text { Ampicillin }\end{array}$ & $7 / 46(15.21 \%)$ & $39 / 46(84.78 \%)$ \\
\hline $\begin{array}{l}\text { 2.b-lactam/b-lactamase-inhibitor combination } \\
\text { Piperacillin+tazobactum }\end{array}$ & $40 / 46(86.95 \%)$ & $06 / 46(13.04 \%)$ \\
\hline $\begin{array}{c}\text { 3.Cephalosporins } \\
\text { Ceftriaxone } \\
\text { Ceftazidime }\end{array}$ & $\begin{array}{l}21 / 46(45.65 \%) \\
19 / 46(41.30 \%)\end{array}$ & $\begin{array}{l}25 / 46(54.34 \%) \\
27 / 46(58.69 \%)\end{array}$ \\
\hline $\begin{array}{l}\text { 4.Carbapenems } \\
\text { Imipenem }\end{array}$ & $46 / 46(100.00 \%)$ & Nil \\
\hline $\begin{array}{c}\text { 5.Aminoglycosides } \\
\text { Amikacin } \\
\text { Gentamicin }\end{array}$ & $\begin{array}{l}35 / 46(76.08 \%) \\
34 / 46(73.91 \%)\end{array}$ & $\begin{array}{l}11 / 46(23.91 \%) \\
12 / 46(26.09 \%)\end{array}$ \\
\hline $\begin{array}{l}\text { 6.Flouroquinolones } \\
\text { Ciprofloxacin }\end{array}$ & $32 / 46(69.56 \%)$ & $14 / 46(30.43 \%)$ \\
\hline $\begin{array}{l}\text { 7.Folate pathway inhibitors } \\
\text { Cotrimoxazole }\end{array}$ & $7 / 46(15.21 \%)$ & $39 / 46(84.78 \%)$ \\
\hline
\end{tabular}

Out of 46 isolates, 6/46 (13.04\%) strains were sensitive to all drugs, whereas 21/46(45.65\%) isolates were resistant to three or more antimicrobial agents. Five $(10.87 \%)$ isolates were resistant to all agents except Imipenem (Figure1). 


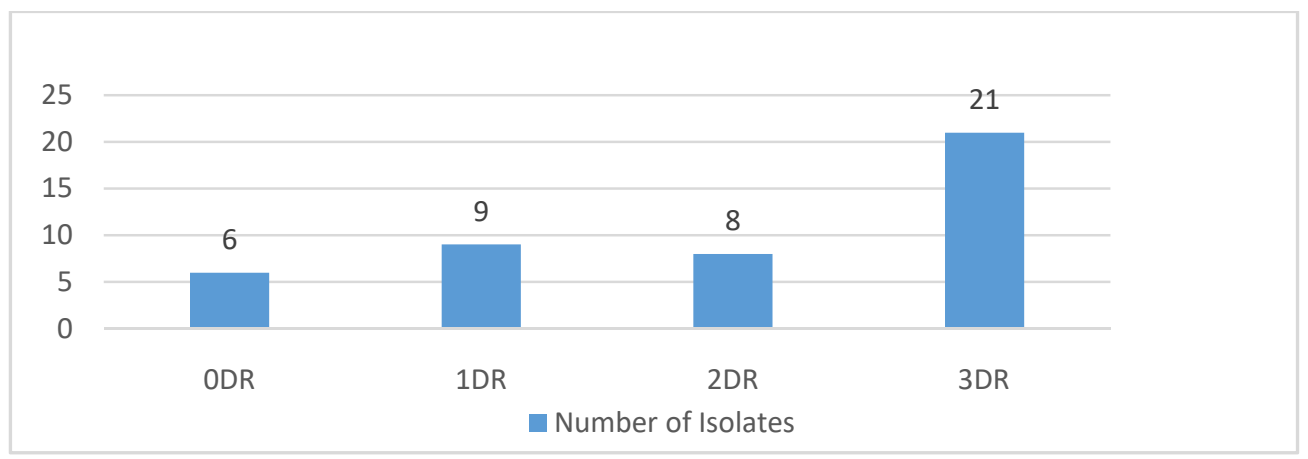

Figure-1: Drug Resistance among E.coli isolates

(0DR- All drugs sensitive/ 1DR-reistant to beta lactams/2DR-resisatnce to beta lactam and folate pathway inhibitors (first line drugs)/ 3DR- resistant to three and $>$ three class of antibiotic s tested)

Resistant pattern of isolates 21/46(45.65\%) which were resistant to three or more than three class of antimicrobial agents is depicted in Table 2.

Table-2: MDR profile of $E$. coli isolates.

\begin{tabular}{|c|c|c|}
\hline Mode of action & Antibiotic class & Resistance (n=21) \\
\hline Inhibition of cell wall synthesis & Beta lactams \\
& Cephalosporin's & $21 / 21(100.00 \%)$ \\
\hline Inhibition of DNA replication & Quinolones & $3 / 21(14.28 \%)$ \\
\hline Inhibition of protein synthesis & Aminoglycosides & $5 / 21(23.80 \%)$ \\
\hline Inhibition of folic acid metabolism & Cotrimoxazole & $21 / 21(100.00 \%)$ \\
\hline
\end{tabular}

\section{Discussion}

Bacteria possess a remarkable ability to develop and acquire antimicrobial resistance. Among GNB, common mechanisms include modification of drug targets; production of inactivating enzymes, such as b-lactamases and aminoglycoside-modifying enzymes; efflux pumps and alterations in outer membrane proteins that are associated with decreased permeability to antibiotics Thus, antibiotic exposure not directly induce these resistance mechanisms. Rather, they promotes proliferation of antibiotic-resistant GNB by exerting selective pressure [9].

Numerous clinical studies have documented the emergence of resistant in association with use of drugs for treatment of pathogens [19-22]. Although our study focuses on the intestinal tract as a site for emergence of antibiotic-resistance, though resistance among GNB also emerge frequently from other sites.

Indeed, members of family Enterobacteriaceae are widely distributed in the environment and cause a variety of infections in hospital and community settings. E. coli, a member of the indigenous colonic microflora, is normally maintained at relatively low population densities by the predominant anaerobic microflora [23].

However, E. coli though harmless intestinal inhabitant, it can also be a highly versatile and frequently deadly pathogen due to its high genetic flexibility to acquire and/or transfer resistance or virulence genes from or to other strains. This problem was more complicated through the emergence of MDR strains to 3 or even more classes of antibacterial agents. The occurrence of MDR is very common and mainly in Gram negative bacteria. In this sense, MDR clinical isolates of E.coli pathogenic strains are commonly seen in clinics represents a major health care problem with increased morbidity and mortality [16]. 
Of the 111 stool samples, 46 E.coli strains were isolated and their susceptibility pattern against 09 antimicrobial agents was studied by disc diffusion method as per CLSI guidelines [17]. We observed decreased susceptibility of E.coli isolates to first line antibiotics (ampicillin: 15.21\% and cotrimoxazole 15.21\%), which tend to be more affordable and accessible to families, followed by $3^{\text {rd }}$ generation cephalosporin's (ceftriaxone $45.65 \%$, ceftazidime $41.30 \%$ ). However, resistance among quinolones and aminoglycosides varies between 20-30\%). Carbapenems (Imipenem) were observed as most sensitive followed by Piperacillin+Tazobactum (100.00\% and $86.95 \%$ respectively) (Table 1). Our study depicted relatively high resistance rates as compared to results by Shakya et al [24] and Cho Sh et al [25].

Table-3: Antimicrobial resistance pattern as observed in other studies.

\begin{tabular}{|c|c|c|c|c|c|c|}
\hline Author \& year & Amp & CTX & CIP & AK & COT & IMI \\
\hline Shakaya et $\mathrm{al}^{24}, 2013$ & $37 \%$ & $13 \%$ & $8 \%$ & $5 \%$ & $29 \%$ & Nil \\
\hline Cho SH et al ${ }^{25}, 2011$ & $76.5 \%$ & $13.6 \%$ & - & $2.1 \%$ & $44.8 \%$ & Nil \\
\hline Malakar M et al & & & & & \\
\hline Present Study & $92 \%$ & $85 \%$ & $31 \%$ & $8 \%$ & $92 \%$ & Nil \\
\hline
\end{tabular}

(AMP-Ampicillin, CTX-Ceftriaxone, CIP-Ciprofloxacin, AK-Amikacin, COT-Cotrimoxazole, IMI-Imipenem)

Our findings were in concordance with Pathania et al [27], who also observed high rate of resistance among E.coli isolates to ampicillin, Cotrimoxazole and cephalosporin's. This can be explained as E.coli being common gastrointestinal isolate, strains are exposed to different antimicrobial agents often used widely and injudiciously.

Among E.coli isolates 21/46(45.65\%) were resistant to three or more than three class of antimicrobials agents. All these isolates were resistant to ampicillin and cotrimoxazole whereas 5/21(23.80\%) and 3/21(14.28\%) were resistant to aminoglycosides and quinolones respectively (Table 2). Five isolates were resistant to eight antibiotics (AMP/CTX/AK/CIP/COT/CEF/GEN/ PcTZ) (Figure 1). Similar results were observed by Cho SH et al [25] with little variations, who observed $78.8 \%$ isolates were resistant to two or more antibiotics and $46 \%$ isolates were MDR.

Antibiotic exposure is not thought to directly induce resistance mechanisms, rather they promotes proliferation of antibiotic-resistant GNB by exerting selective pressure and facilitate emergence of new resistant mutants or of preexisting subpopulations of resistant organisms [18].

Several factors have contributed to the current state of the crisis which include: Inappropriate use of antibiotics in clinical practice and animal industry, poor infection control measures, poor sanitation and infrastructure, decreased access to clinical microbiology laboratory and data, lack of research in clinical infectious diseases especially controlled randomized trials, long courses of antibiotics, evolution of bugs and microbiomes, increased immunocompromised states (i.e., due to older population, HIV epidemic, new chemotherapeutics including immune-modulating agents), globalization, and increased travel among others [28].

Antibacterial resistance is complex and dynamic. Although the major genetic and biochemical mechanisms have long been recognized, new factors continue to be discovered, including integrons, multidrug efflux, hyper mutability, and plasmid addiction. Within many individual isolates, the complexity of resistance is increasing, with multiple determinants carried, and with genes being gained, amplified, and lost [2].

Limitation of our study- Although studies of healthy volunteers usually provide a useful reference regarding the potential impact of antibiotics on colonization with gram-negative bacilli. Our study group included patients who are at increased risk of exposure to exogenous antibiotic-resistant GNB. Also, oropharynx of hospitalized patient frequently becomes colonized with GNB that may be ingested. Colonic microflora of ill hospitalized patients may be altered in the absence of antibiotic therapy. Finally, patients often receive multiple antibiotics concurrently or in sequence, which makes it difficult to determine the effects of individual agents. Thus limiting it's applicability to clinical settings. 
To conclude, relatively high antibiotic resistance as well as MDR were detected in the isolated E. coli strains. Thus, intestinal $E$. coli has undergone a remarkable transformation in recent decades and undoubtedly will continue to evolve. Once dismissed as a harmless inhabitant of the intestinal tract, E. coli is now seen as a pathogenic species with remarkable versatility in its ability to cause disease in humans and animals. This dynamism promises to present new challenges in the treatment and prevention of $E$. coli infections. Therefore, adherence to standard infection control measures and good antibiotic stewardship are essential control strategies. We also suggest periodic surveillance for resistant strains and rational use of antimicrobial agents is necessary for effective treatment and prediction of occurrence of resistant populations.

\section{Funding: Nil, Conflict of interest: None Permission of IRB: Yes}

\section{References}

1. Calva JJ, Osorino JS, Ceron C. Antimicrobial resistance in fecal flora: longitudinal community based surveillance of children from urban Mexico. Antimicrobial agents \& chemotherapy. 1996 Jul;40(7): 1699-1702.

2. Livermore DM. Bacterial resistance: origins, epidemiology, and impact. Clin Infect Dis. 2003 Jan 15; 36 (Suppl 1):S11-23.

3. Bridges BA. Hypermutation in bacteria and other cellular systems. Philos Trans R Soc Lond B Biol Sci. 2001 Jan 29;356(1405):29-39.

4. Poole K. Multidrug efflux pumps and antimicrobial resistance in Pseudomonas aeruginosa and related organisms. J Mol Microbiol Biotechnol. 2001 Apr;3(2): 255-64.

5. Cohen SP, McMurry LM, Hooper DC, Wolfson JS, Levy SB. Cross-resistance to fluoroquinolones in multiple-antibiotic-resistant (Mar) Escherichia coli selected by tetracycline or chloramphenicol: decreased drug accumulation associated with membrane changes in addition to OmpF reduction. Antimicrob Agents Chemother 1989 Aug;33(8):1318-25

6. Livermore DM. Antibiotic resistance in staphylococci. Int J Antimicrob Agents. 2000 Nov; 16 Suppl 1: S3-10.

7. Hall RM. Mobile gene cassettes and integrons: moving antibiotic resistance genes in gram-negative bacteria. Ciba Found Symp. 1997; 207: 192-202; discussion 202-5.

8. Bauernfeind A, Chong Y, Lee K. Plasmid-encoded AmpC beta-lactamases: how far have we gone 10 years after the discovery? Yonsei Med J. 1998 Dec;39 (6):520-5.

9. Oliver A, Perez-Diaz JC, Coque TM, Baquero F, Canton R. Nucleotide sequence and characterization of a novel cefotaxime-hydrolyzing b-lactamase.(CTX M10) isolated in Spain. Antimicrob Agents Chemother 2001 Feb; 45(2):616-20. DOI:10.1128/AAC.45.2.616.620.2001 .

10. Livermore DM. beta-Lactamases in laboratory and clinical resistance. Clin Microbiol Rev. 1995 Oct;8 (4):557-84.

11. Carlet J. The gut is epicenter of antibiotic resistance. Antimicrobial resistance and infection control. 2012 Nov; 1: 39. DOI: 10.1186/2047-2994-1-39.

12. Rasheed MU, Thajuddin N, Ahamed P, Teklemariam Z, Jamil K. Antimicrobial drug resistance in strains of Escherichia coli isolated from food sources. Rev Inst MedTrop Sao Paulo.2014Jul-Aug;56(4):341-6.

13. Murray BE. Problems and dilemmas of antimicrobial resistance. Pharmacotherapy. 1992;12(6 Pt 2):86S-93S.

14. Collee JG, Duguid JP, Fraser AG, Marmion B P, Simmons A. Laboratory strategy in the diagnosis of infective syndrome. In Collee JG, Fraser AG, Marmion BP, Simmons A, editors. Mackie \& McCartney Practical Medical Microbiology. 14 ${ }^{\text {th }}$ ed. New Delhi: Elsevier, a division of Reed Elsevier India Pvt. Ltd. 2006. P.53-94.

15. Katouli M. Population structure of gut Escherichia coli and its role in development of extra-intestinal infections. Iran J Microbiol. 2010 Jun;2(2):59-72. 
16. Aly MEA, Essam TM, Amin MA. Antibiotic resistance profile of E.coli strains isolated from clinical specimens and food samples in Egypt. Intl $\mathrm{J}$ Microbiol Res.2012;3(3):176-82.

17. Wayne PA. Clinical and laboratory standard institute 2006. Performance standards for antimicrobial disc tests. Approved Standards, 9th ed.; sixteenth informational supplement M2-M9. 2006;26.

18. Donskey CJ. Antibiotic regimen and intestinal colonization with antibiotic resistant gram negative bacilli. Antibiotics and gram negative bacilli. CID. 2006 Sep;43(S2):S64-9. DOI:10.1086/504481.

19. Peña C, Pujol M, Ardanuy C, Ricart A, Pallares R, Liñares J, Ariza J, Gudiol F. Epidemiology and successful control of a large outbreak due to Klebsiella pneumoniae producing extended-spectrum betalactamases. Antimicrob Agents Chemother. 1998 Jan;42 (1): 53-8.

20. Olson B, Weinstein RA, Nathan C, Chamberlin W, Kabins SA. Epidemiology of endemic Pseudomonas aeruginosa: why infection control efforts have failed. J Infect Dis. 1984 Dec;150(6):808-16.

21. Lucet JC, Decré D, Fichelle A, Joly-Guillou ML, Pernet M, Deblangy C, Kosmann MJ, Régnier B. Control of a prolonged outbreak of extended-spectrum beta-lactamase-producing enterobacteriaceae in a university hospital. Clin Infect Dis. 1999 Dec;29 (6): 1411-8.

22. Wiener J, Quinn JP, Bradford PA, Goering RV, Nathan C, Bush K, Weinstein RA. Multiple antibioticresistant Klebsiella and Escherichia coli in nursing homes. JAMA. 1999 Feb 10;281(6):517-23.
23. Freter R, Brickner H, Fekete J, Vickerman MM, Carey KE. Survival and implantation of Escherichia coli in the intestinal tract. Infect Immun. $1983 \mathrm{Feb}$;39 (2): 686-703.

24. Shakya P, Barett P, Diwan V, Marothi Y, Shah H, Chhari N, et al. Antibiotic resistance among $\mathrm{E}$ coli isolates from stool samples of children aged 3 to 14 years from Ujjain, India. BMC Infectious diseases. 2013 Oct; 13: 477-83. DOI: 10. 1186/1471-233413-477.

25. Cho SH, Lim Sl, Park SL, Kim SH, Kang YE. Prevalence of antibiotic resistance in Escherichia coli fecal isolates from healthy patients and patients with diarrhoea. Public health Res Prespect. 2011;2(1):415.Doi-10.1016/j.phrp.2011.05.003.

26. Malakar M. Antimicrobial susceptibility pattern of Escherichia coli isolated from urine and stool samples of infected patients in Lakihmpur district of Assam, India. International Journal of Innovative Science, Engineering \& technology.2014;1(2):184-9.

27. Rathaur VK, Pathania M,Jayara A,Yadav N. Clinical study of acute childhood diarrhea caused by bacterial Enteropathogens. J Clin Diag Res.2014;8 (5): PC) 01-5.DOI:10.7860/JCDR/2014/6677.4319.

28. Hassani M. The Crisis of Resistant Gram Negative Bacterial Infection: Is there any Hope for ESKAPE? Clin Res Infect Dis.2014;1(1):1005-9.

29. Hooper DC. Emerging mechanisms of fluoroquinolone resistance. Emerg Infect Dis. 2001 Mar-Apr; 7 (2):337-41.

\section{How to cite this article?}

Sayal P, Kumar S. Antimicrobial resistance pattern of faecal Escherichia coli isolates: story of antibiotic use and misuse. Int J Med Res Rev 2017;5(04):386-392 doi:10.17511/ijmrr. 2017.i04.03. 